\title{
Calculation of phase angle diversity for time-varying harmonic currents from grid measurement
}

\author{
A.M. Blanco, J. Meyer and P. Schegner \\ Institute of Electrical Power Systems and High Voltage Engineering \\ Technische Universitaet Dresden \\ Mommsenstraße 10, 01062 Dresden (Germany) \\ Phone/Fax number: +0049 351 463-32217, e-mail: ana.blanco@tu-dresden.de
}

\begin{abstract}
Phase angle diversity of harmonic currents emitted by different equipment, like electronic power supplies, modern lamps with electronic ballast, chargers for electrical cars or photovoltaic inverters, results in cancellation effects which represent an efficient way of managing harmonic levels in LV grids. Different methods have been presented in the last decades to quantify and evaluate the summation of harmonic currents based on grid measurements. There is not a general consensus about which method or index should be preferred, especially if time-variation of harmonic currents is considered. The paper discusses the impact of measurement accuracy and aggregation parameters on the calculation of the two main diversity indices, namely diversity factor and summation exponent. In case of timevarying harmonics an additional statistical post-processing is required which also depends on several parameters and calculation methods. Based on the results of the application of different methods to real grid measurement data, some guidance on suitability and limitations of the different methods are given.
\end{abstract}

\section{Keywords}

Time-varying harmonics, diversity factor, summation exponent, aggregation methods

\section{Introduction}

Number and variety of non-linear devices and installations used by residential and commercial customers are increasing continuously. Therefore the effect of harmonics in the network is more and more considered in both the planning and the operation of distribution systems. This analysis is complex, because multiple factors influence the emission and propagation of harmonics through the network, like the network impedance, the voltage distortion and the time-variation of number and type of connected equipment.

One of the key aspects of a realistic harmonic analysis is a correct representation of summation of harmonic currents. The presence of different devices with different topologies at one connection point can cause a diversity of current harmonic phase angles and subsequently may lead to a lower magnitude of vector sum than the arithmetical sum of the harmonic currents [1]. This is known as diversity effect (or cancellation effect) and has a high influence on the total harmonic distortion emitted by larger groups of non-linear loads into the grid. To quantify this effect commonly two different indices are used: summation exponent and diversity factor.

Most of the papers that address the diversity of harmonic currents consider only the effect of few devices in a single moment of time or a perfect steady state of harmonics [e.g. [2-4]). Variation of system, load and generation result in a time-varying harmonic currents and an additional statistical post-processing (like for harmonic assessment according to EN 50160) is required in order to calculate aggregated diversity indices. Parameters and methods used for this post-processing may have a considerable impact on the calculated diversity index and are not yet defined. Furthermore, the accuracy of these indices depends on the accuracy of the measurement instrument. Inaccuracies of the current input channels may cause differences between vector sum and total current, which finally can result in erroneous diversity indices. If more than a single measurement instrument is required, also clock synchronization between the instruments may affect the accuracy of the diversity index calculation.

The main aim of the paper is a systematic analysis of those issues in order to identify a most suitable method for an accurate and reliable measurement and assessment of summation effects of time-varying harmonic currents in public LV grids. The analysis in this paper is based on measurement data of a typical MV/LV substation with residential and commercial customers connected.

The first part of the paper describes in detail the example measurement data used for the analysis and provides the definition of the diversity indices. Next chapter is focused on the impact of measurement uncertainty and clock synchronization on the accuracy of the diversity index calculation. The last chapter discusses the different methods for calculating diversity indices under time-varying conditions as well as the impact of the statistical post-processing parameters on the results.

\section{Measurement setup}

One MV/LV substation of a residential area was selected for the measurements. The substation has a $630 \mathrm{kVA}$ transformer which supplies different residential and commercial loads by 6 feeders. The feeders have different lengths and the load is not uniformly distributed 


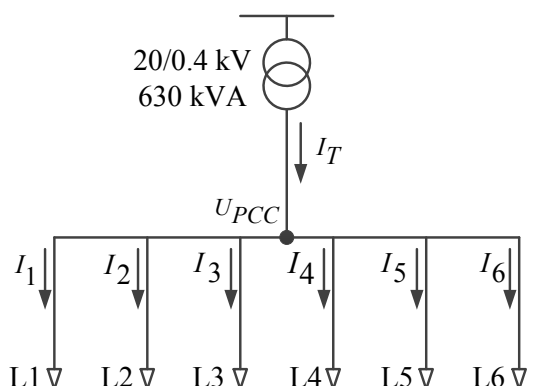

Fig. 1. Layout of the measured MV/LV substation

along it. The general schema of the layout is shown in Fig. 1. Table I details the number and type of users and the length of the lines.

Table I. Characteristics of the selected MV/LV substation

\begin{tabular}{|c|c|c|c|c|}
\hline \multirow{2}{*}{ Feeder } & \multirow{2}{*}{ Length } & \multicolumn{3}{|c|}{ Number of users } \\
\cline { 3 - 5 } & & Households & Offices & Stores \\
\hline L1 & 380 & 99 & 1 & 0 \\
\hline L2 & 60 & 32 & 2 & 3 \\
\hline L3 & 510 & 114 & 2 & 7 \\
\hline L4 & 190 & 50 & 1 & 3 \\
\hline L5 & 325 & 42 & 20 & 4 \\
\hline L6 & 655 & 112 & 13 & 8 \\
\hline Total & 2120 & 449 & 39 & 25 \\
\hline
\end{tabular}

A network analyzer which allows exact synchronized measurements and stores gapless 10-period-values according to the standard IEC 61000-4-30 [5] was used for the measurements. Rogowski coils were used for the current measurements. The accuracy of the current measurement depends mainly on the accuracy of the coils, which is better than $\pm 1 \%$ for currents higher than $0.3 \mathrm{~A}$ according to the manufacturer. However, this accuracy is too conservative and usually smaller currents can be accurately measured. The phase angle error is smaller than $\pm 1.4^{\circ}$.

The voltage at the LV busbar $\left(U_{P C C}\right)$, the total current in the main feeder $\left(I_{T}\right)$ and all feeder currents $\left(I_{1} \ldots I_{6}\right)$ were measured during one week. The magnitudes and angles of voltage and current harmonics up to $50^{\text {th }}$ order, the active and apparent power and the total voltage and current distortion (THD) were recorded. Due to the limited number of available input channels and the requirement of exact time clock synchronization, it was not possible to measure the three phases of all currents at the same time. Therefore, each phase was measured individually for two consecutive days (about 850000 measurement values per phase).

Fig.2a shows exemplarily the load curves of the complete substation (main feeder) of the three phases for a single day. The curves of phases $\mathrm{A}$ and $\mathrm{C}$ correspond to two different workdays (Wednesday and Tuesday respectively) while the curve of phase B corresponds to a Saturday. During the workdays the consumption is higher between $8 \mathrm{am}$ and $6 \mathrm{pm}$ due to the high amount of offices and stores. During the weekend the load curve has two peaks at noon and $7 \mathrm{pm}$, which corresponds to the typical behavior of residential users.

The harmonic emission also varies during the day. Fig. $2 b$ shows the variation of magnitude and phase angle of the 3rd harmonic in the complex plane for all feeders and phase A. Phases B and C behave similar. The similarity of harmonic magnitudes and angles between the feeders is due to the similar loads in each feeder. However, this similarity can only be observed for $3^{\text {rd }}, 5^{\text {th }}$, and $7^{\text {th }}$ harmonics. Higher order harmonics show higher variations of magnitudes and angles. Exemplarily Fig. 2c presents the measurement of $17^{\text {th }}$ harmonic in the complex plane. The angle of $17^{\text {th }}$ harmonic has a high variation (more than $180^{\circ}$ ) while $3^{\text {rd }}$ harmonic shows only a low variation between 130 and $210^{\circ}$. Actually, for the $3^{\text {rd }}$ harmonic a prevailing phase angle of approximately $200^{\circ}$ can be adopted, while for $17^{\text {th }}$ harmonic a prevailing phase angle is more difficult to define.

\section{Calculation of diversity indices}

Two different indices are commonly used for the quantification of the diversity effect: diversity factor and summation exponent. The diversity factor $k_{p}^{(h)}$ for an individual harmonic $h$ is calculated by [3]:

$$
k_{p}^{(h)}=\frac{\text { Vector } \text { sum }}{\text { Arithmeticsum }}=\frac{I_{V E C}^{(h)}}{I_{A R I}^{(h)}}=\frac{\left|\sum_{i=1}^{n} \underline{I}_{i}^{(h)}\right|}{\sum_{i=1}^{n}\left|I_{i}^{(h)}\right|}
$$

where $\underline{I}_{i}^{(h)}$ represents the harmonic current vector of an individual device or feeder $i, n$ is the number of devices or feeders and $h$ the order of the harmonic. The diversity factor varies between 0 (perfect cancellation) and 1 (no cancellation). This index can be calculated only if current

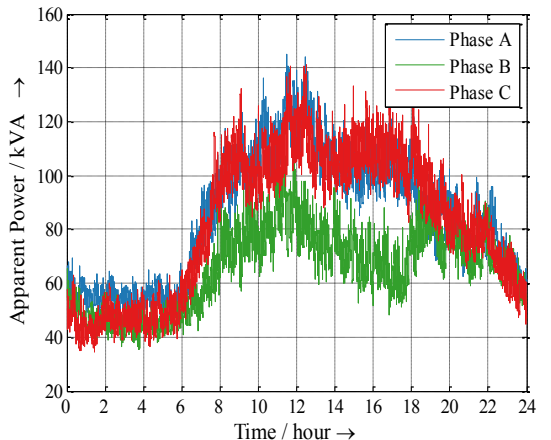

a)

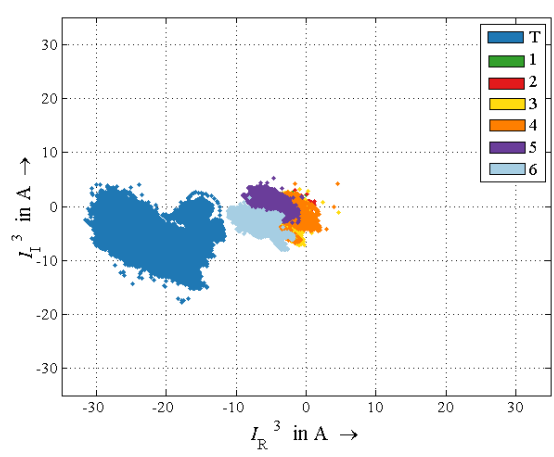

b)

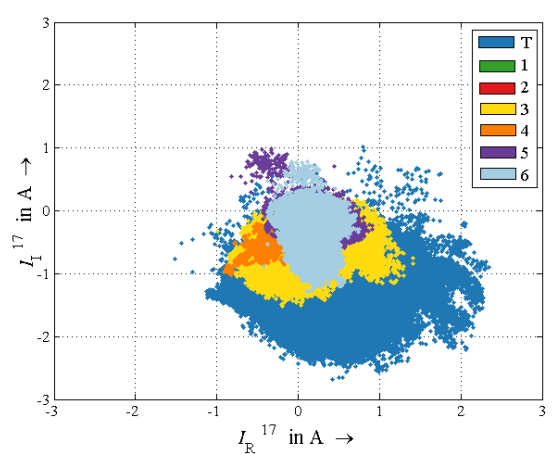

c)

Fig. 2. a) Apparent power during single days in phases $A, B$ and $C$ of the main feeder (10 period values)

b) 3rd harmonic currents of all feeders during a single day in the complex plane (phase A)

c) 17 th harmonic currents of all feeders during a single day in the complex plane (phase A) 
harmonic magnitudes and angles of all feeders are known.

The summation exponent $\alpha^{(h)}$ is determined by solving the following non-linear equation iteratively:

$$
I_{V E C}^{(h)}=\alpha \sqrt{\sum_{i=1}^{n}\left|\underline{I}_{i}^{(h)}\right|^{\alpha}}
$$

This index can even be calculated without the harmonic phase angles information, if the current harmonic magnitudes of the main feeder and all individual feeders are known. A summation exponent $\alpha^{(h)}=1$ correspond to $k_{p}^{(h)}=1$ and represents the arithmetic sum of the currents (no cancellation). If $\alpha^{(h)}=2$, the vector sum is equal to the root sum of squares of the current harmonic magnitudes. The higher the value of $\alpha^{(h)}$, the better is the cancellation between the current harmonics. In case of perfect cancellation the summation exponent would become infinite. The relation between $\alpha^{(h)}$ and the level of cancellation is not linear and under some conditions the equation may not have a solution.

The technical report IEC 61000-3-14 [6] use the index $\alpha^{(h)}$ to consider different diversity behavior of different harmonic orders. The technical report gives some reference values of $\alpha^{(h)}$ for planning studies, which can be adopted in absence of specific information:

Table II. Summation exponent for harmonics [6]

\begin{tabular}{|c|c|}
\hline Harmonic order & $\alpha^{(h)}$ \\
\hline$h<5$ & 1 \\
\hline $5 \leq h \leq 10$ & 1,4 \\
\hline$h>10$ & 2 \\
\hline
\end{tabular}

Due to its different ranges, levels of non-linearity and sensitivity, both indices are always in parallel discussed in the paper.

Diversity factor and the summation exponent are defined for a single time instant. The calculation of a single value for these indices in case of measurements containing more than one time instant requires a statistical postprocessing which needs the specification of the following additional parameters:

1) Aggregation interval (e.g. 1 minute, 10 minute, ...),

2) Aggregation method (e.g. Maximum values, RMS-values, ...),

3) Observation interval (e.g. 1 day, 1 week, ...)

4) Assessment percentile (e.g. 95-\%-percentile, 99-\%-percentile, ...)

5) Statistical calculation method (e.g. based on current c.d.f.'s, based on diversity index c.d.f., ...)

In case of perfect steady state conditions, the diversity indices are independent of the above mentioned parameters. In case of time-varying harmonics, which are common for most feeder measurements, the parameters can have a significant impact on the calculated diversity indices which is discussed in detail in chapter 5 .

\section{Impact of measurement accuracy}

\subsection{Accuracy of measured harmonic currents}

In order to determine the diversity factors or summation exponents, the measured currents should be accurate and time-synchronized. Due to the simultaneous measurements of all feeders, the data set is always exact time synchronized. To verify accuracy and usability of the measured data, the total harmonic current is calculated by the vector sum of the 6 feeders for each time instant. In case of a reliable measurement, the difference between calculated total current and measured total current should be reasonable small.

Fig. 3 shows the $99^{\text {th }}$ percentile of the absolute errors for selected harmonics and all phases. Introducing a maximum allowable error of about $5 \%$, all odd harmonics from $3^{\text {rd }}$ to $17^{\text {th }}$ order excluding $11^{\text {th }}$ order are suitable for further analysis. The excluded harmonics have very low magnitudes below the threshold of the measurement system, which explains the high difference between calculated and measured currents.

The above described method for selecting harmonics for further analysis should be always applied in advance in order to ensure reliable results. To get a better idea of the impact of measurement accuracy and clocksynchronization on diversity indices calculation, a sensitivity analysis is carried out for both impact factors based on synthetic measurement data.

\subsection{Impact of measurement accuracy}

A synthetic network with 6 feeders and 8 different load scenarios is used for the analysis. The selected load scenarios consider the connection of disturbing devices of different size and type in each feeder. Therefore, for each scenario different prevailing harmonic magnitudes and angles are defined per feeder (Table III). Those values represent the exact measured values. E.g. for the first scenario the harmonics in each feeder is approximately equal with small magnitude variation between 5 and $7 \mathrm{~A}$ and small phase angle variations between 0 and $90^{\circ}$. The first scenario group ( 1 to 4 ) considers low variation of the harmonic magnitudes between the feeders, whereas the second scenario group ( 5 to 8 ) considers a high variation (between 2 and 10A). Within each group the phase angle variation increases between the scenarios.

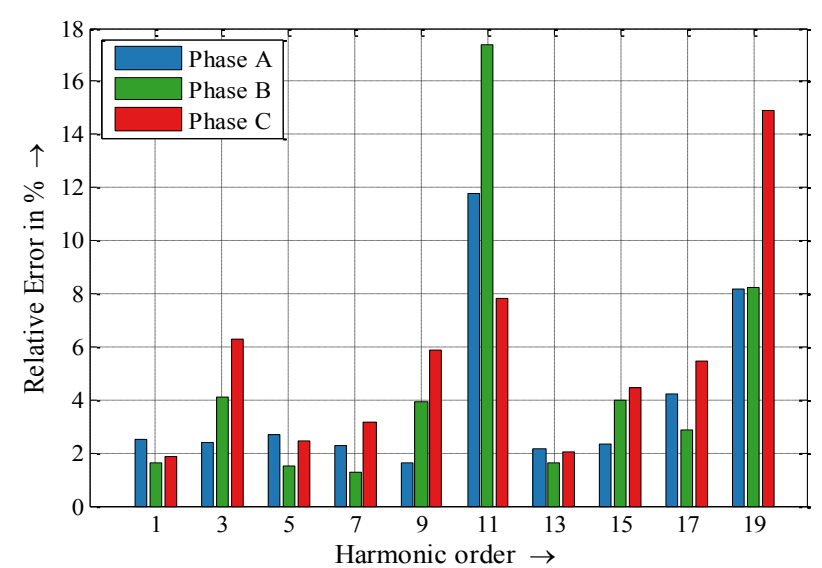

Fig. 3. 99th percentile of the relative errors between vector sum of the feeder's currents and measured current $I_{T}$ 
Table III. Exact harmonic magnitudes and angles of the feeders for each load scenario

\begin{tabular}{|c|c|c|c|c|c|c|c|}
\cline { 2 - 8 } \multicolumn{1}{c|}{} & \multirow{2}{*}{ Scenario } & \multicolumn{6}{|c|}{ Feeder } \\
\cline { 2 - 8 } & 1 & 2 & 3 & 4 & 5 & 6 \\
\hline \multirow{2}{*}{$|\mathrm{I}|$ in A } & $1-4$ & 5.2 & 5.5 & 5.8 & 6.4 & 6.5 & 7 \\
\cline { 2 - 8 } & $5-8$ & 2.5 & 3.2 & 4.1 & 6.8 & 7.2 & 9.4 \\
\hline \multirow{4}{*}{$\angle \mathrm{I}$ in $\circ$} & 1,5 & 30 & 10 & 25 & 65 & 72 & 50 \\
\cline { 2 - 8 } & 2,6 & 30 & 60 & 90 & 120 & 150 & 170 \\
\cline { 2 - 8 } & 3,7 & 30 & 60 & 260 & 120 & 150 & 200 \\
\cline { 2 - 8 } & 4,8 & 30 & 80 & 100 & 160 & 235 & 300 \\
\hline
\end{tabular}

Based on the exact measurement values in Table III, the exact diversity indices are calculated and shown in Table IV.

Table IV. Exact diversity indices for the different scenarios

\begin{tabular}{|c|c|c|c|c|c|c|c|c|}
\hline \multirow{2}{*}{ Indices } & \multicolumn{10}{|c|}{ Scenario } \\
\cline { 2 - 10 } & 1 & 2 & 3 & 4 & 5 & 6 & 7 & 8 \\
\hline $\mathrm{k}_{\mathrm{P}}$ & 0.93 & 0.67 & 0.33 & 0.13 & 0.94 & 0.73 & 0.5 & 0.15 \\
\hline $\mathrm{A}$ & 1.04 & 1.28 & 2.66 & -- & 1.04 & 1.22 & 1.73 & -- \\
\hline
\end{tabular}

The measurement error of each input channel is represented by two normal distributions for magnitude uncertainty and phase angle uncertainty. While the mean values $(\mu)$ of the distribution functions correspond to the exact values given in Table III, the standard deviation $(\sigma)$ is calculated based on accuracies of typical measurement instruments given in Table V. It is assumed that the provided accuracy corresponds to three times the standard deviation. Three different cases are defined with different standard deviations considering a high, a normal and a low accuracy. Case 2 corresponds to the uncertainty of the measurement instrument used in this project.

Table V. Standard deviations for the different accuracy cases.

\begin{tabular}{|c|c|c|c|c|}
\hline & \multicolumn{2}{|c|}{ Magnitudes } & \multicolumn{2}{c|}{ Angles } \\
\hline Case & Accuracy & $\sigma$ & Accuracy & $\sigma$ \\
\hline 1 & $\pm 0.16 \mathrm{~A}$ & $0.055 \mathrm{~A}$ & $\pm 0.7^{\circ}$ & $0.25^{\circ}$ \\
\hline 2 & $\pm 0.3 \mathrm{~A}$ & $0.11 \mathrm{~A}$ & $\pm 1.4^{\circ}$ & $0.5^{\circ}$ \\
\hline 3 & $\pm 0.64 \mathrm{~A}$ & $0.22 \mathrm{~A}$ & $\pm 2.8^{\circ}$ & $1^{\circ}$ \\
\hline
\end{tabular}

In a next step, 1000 values of harmonic current magnitudes and phase angles of each feeder were sampled per load scenario and accuracy case. Fig. 4 exemplarily shows the distribution function (CDF) of magnitude and phase angle for scenario $1 /$ case 1 . For each of the 1000 sample sets the indices $k_{P}$ and $\alpha$ are calculated and the difference between calculated and exact index (Table IV) is calculated. Fig. 5 presents this difference as percentage value related to the respective exact index for all scenarios and accuracy cases. It was not possible to calculate $\alpha$ in scenarios 4 and 8 due to the high cancelation between the currents (variation of the angles between 0 and $360^{\circ}$ ). Scenarios 1 and 5 show almost no variation of diversity indices (less than 1\%), while cases 4 and 8 have a considerable high variation.

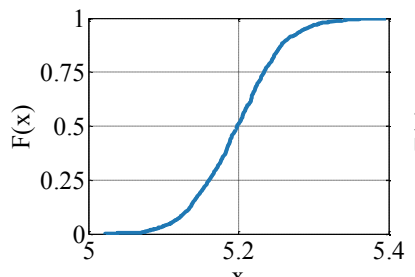

a)

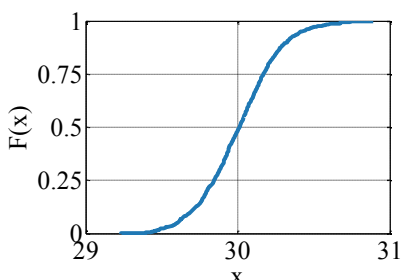

b)
Fig. 4. CDF of harmonic magnitudes (a) and angles (b) of feeder 1 for load scenario 1 and accuracy case 1
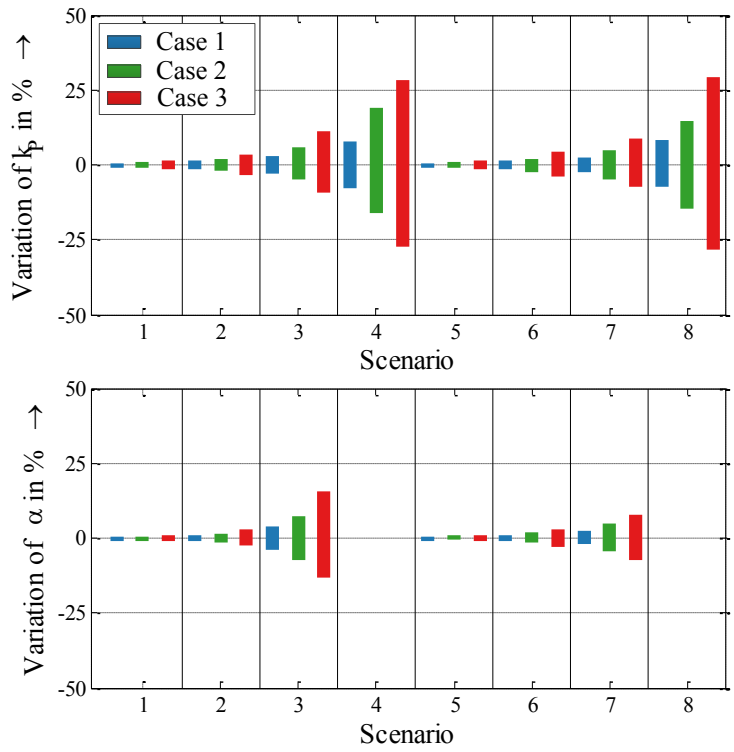

Fig. 5. Variation of $k_{P}$ and $\alpha$ for the selected load scenarios and $\sigma$ cases

As expected, the error in the calculation of diversity indices increases not only with the accuracy of the measurement equipment, but is also strongly linked to the diversity behavior of harmonic phase angles. If the analyzed currents have similar magnitudes and angles within one quadrant, instruments with low accuracies are sufficient. If the currents have higher variations in terms of magnitude but especially of phase angle (spreading in more than 2 quadrants) then only devices with high accuracy should be used. Table III in conjunction with Fig. 5 can give a first guidance.

\subsection{Impact of clock-synchronization}

A part of the real measurement data was used to analyze the impact of possible clock differences on the calculation of diversity indices. The interval between 8a.m. and 11a.m. was selected based on the analysis of the variation between consecutive time instants. It is assumed that an individual measurement instrument is used for each feeder and the total current. The time shift between the internal clocks of the instruments was randomly selected from the following four ranges: $\pm 1 \mathrm{~s}, \pm 10 \mathrm{~s}, \pm 1 \mathrm{~m}$ and $\pm 10 \mathrm{~m}$. IEC $61000-4-30$ requires $\pm 1 \mathrm{~s}$ per day without external synchronization, e.g. by GPS. Applying the time shifts on the measurement data the diversity factors were calculated for each case and two harmonics: $3^{\text {rd }}$ harmonic

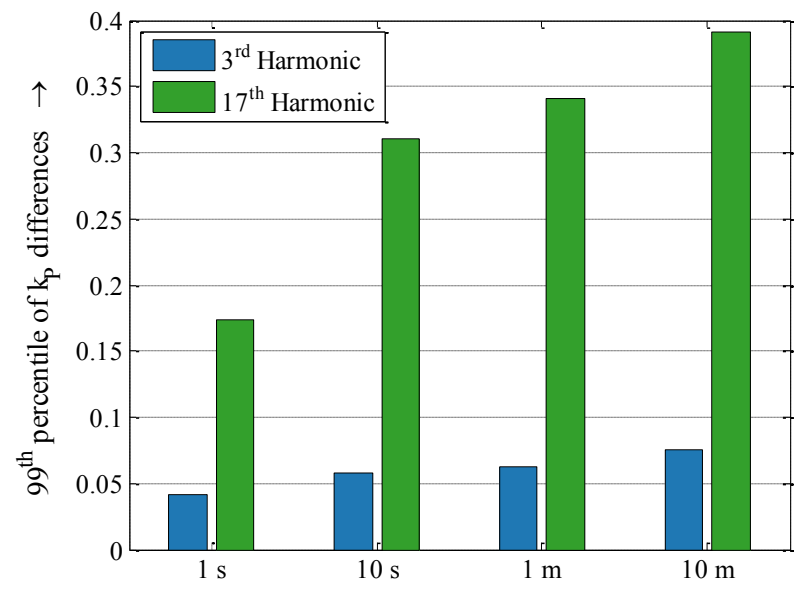

Fig. 6. Differences of $k_{P}$ for different time shifts 
with low diversity and $17^{\text {th }}$ harmonic with high diversity. Finally the difference between the results and the diversity factor without time shift was calculated.

Fig. 6 shows the $99^{\text {th }}$ percentile of the differences in $k_{P}$ for each clock uncertainty. The error increases with increasing uncertainty. While it is negligible small for the $3^{\text {rd }}$ harmonic, it cannot be neglected anymore for the $17^{\text {th }}$ harmonic. Especially in case of higher diversity and higher variation of a harmonic (e.g. caused by more dynamic loads) a higher clock accuracy is required. In case of multiple instruments it is therefore strongly recommended to use GPS synchronization. Only for low order harmonics this may not be necessary.

\section{Impact of statistical post-processing}

Chapter 3 explains the different factors with impact on the calculation of diversity factor and summation exponent in presence of time-varying currents. In this study only the impact of different aggregation intervals (1), assessment percentile (4) and statistical calculation methods (5) is analyzed. According to [5], RMS-aggregation is used as aggregation method (2). The observation interval (3) covers always the whole dataset. As described in cp. 4.1 only odd harmonics from order 3 to 17 excluding the $11^{\text {th }}$ harmonic were considered.

It has to be noted that if percentiles of both diversity indices have to be compared, different percentiles for $k_{P}$ and $\alpha$ have to be used. While $k_{P}$ varies from 1 down to $0, \alpha$ varies from 1 up to infinite. Therefore, the $\mathrm{p}^{\text {th }}$ percentile for $k_{P}$ has to be compared with the $(100 \% \text {-p) })^{\text {th }}$ percentile for $\alpha$.

\subsection{Aggregation Interval}

For the aggregation interval three different cases are distinguished: No aggregation (10 period values), 1-minaggregation and 10-min-aggregation. The aggregation of harmonic magnitudes was performed using the RMSaggregation [5]. No standard exists for the aggregation of harmonic phase angles. Based on common practice, the vector sum of all measurements in the considered aggregation interval was calculated and the phase angle of the resulting vector was used as the aggregated value.

Fig. 7 shows the variation of diversity factors and summation exponents of odd harmonics of phase A. Similar results were obtained for phases B and C. The diversity indices were calculated using Method M1 (cf. to cp. 5.3). Each bar represents the $1^{\text {st }}$ to $99^{\text {th }}$ percentile range

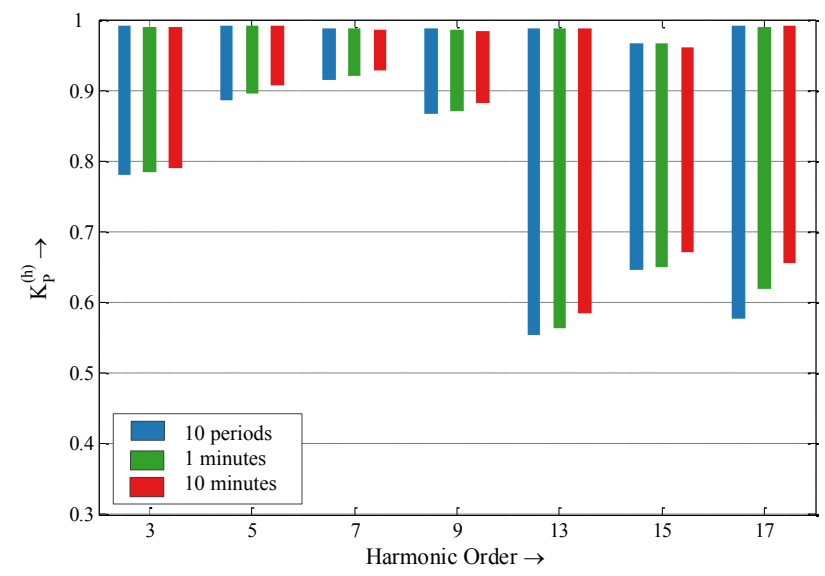

of all diversity indices. The colors represent the different aggregation intervals.

Harmonics up to $9^{\text {th }}$ order show a low aggregation of harmonic currents $\left(k_{P}\right.$ above 0.8 and $\alpha$ below 1.2$)$ due to the similarity of harmonic phase angles. Other harmonics have higher variation of diversity indices and sometimes they have better harmonic cancellations $\left(k_{P}\right.$ below 0.7 and $\alpha$ above 1.4).

The aggregation intervals do not show a significant impact on the results for harmonics up to $9^{\text {th }}$ order. The size of the bars changes slightly for those harmonics and the $99^{\text {th }}$ percentile of $k_{P}$ ( $1^{\text {st }}$ percentile of $\alpha$ respectively) remains practically unaltered. This is in good agreement with the results in [1]. However, the variation seems to increase for higher harmonics. E.g. the $17^{\text {th }}$ harmonic shows a change in the summation exponent of approximately 0.2 units between the results with 10 period-values and 10-min-aggregation. This variation could become considerable for higher harmonics and seems to be linked to the fact that the diversity of harmonic phase angles increases with the order of the harmonic.

\subsection{Assessment percentile}

For assessment percentile the impact of $95^{\text {th }}$ vs. $99^{\text {th }}$ percentiles for $k_{P}$ and $5^{\text {th }}$ vs. $1^{\text {st }}$ percentile for $\alpha$ are analyzed. The percentiles of diversity indices were calculated for each harmonic and each phase. No aggregation was applied to the data and method M1 (cf. to cp. 5.3) was used. The relative error between both percentile pairs (related to the higher percentile) is shown in Table VI. For harmonics below $9^{\text {th }}$ order the difference of assessment percentiles is insignificant (error below $1 \%$ ), but for higher harmonics the difference begins to increase (highlighted values). The diversity indices seem to be more sensitive to higher phase angle diversity.

Table VI. Relative error between assessment percentiles of diversity factors. Values in percent

\begin{tabular}{|c|c|c|c|c|c|c|c|c|}
\cline { 2 - 9 } \multicolumn{1}{c|}{} & \multirow{2}{*}{ Phase } & \multicolumn{7}{|c|}{ Harmonic Order $h$} \\
\cline { 2 - 9 } \multicolumn{1}{c|}{} & & 3 & 5 & 7 & 9 & 13 & 15 & 17 \\
\hline \multirow{3}{*}{$\alpha^{(h)}$} & $\mathrm{A}$ & 0.3 & 0.3 & 0.3 & 0.6 & 0.9 & 1.3 & 1.3 \\
\cline { 2 - 9 } & $\mathrm{B}$ & 0.1 & 0.2 & 0.5 & 0.3 & 0.3 & 0.6 & 0.7 \\
\cline { 2 - 9 } & $\mathrm{C}$ & 0.1 & 0.2 & 0.2 & 0.2 & 1.3 & 2.7 & 2.7 \\
\hline \multirow{3}{*}{$k_{p}^{(h)}$} & $\mathrm{A}$ & 0.6 & 0.6 & 0.4 & 1.0 & 1.4 & 2.1 & 1.9 \\
\cline { 2 - 9 } & $\mathrm{B}$ & 0.2 & 0.3 & 0.8 & 0.4 & 0.5 & 0.8 & 1.0 \\
\cline { 2 - 9 } & $\mathrm{C}$ & 0.2 & 0.4 & 0.3 & 0.3 & 1.7 & 3.5 & 3.1 \\
\hline
\end{tabular}

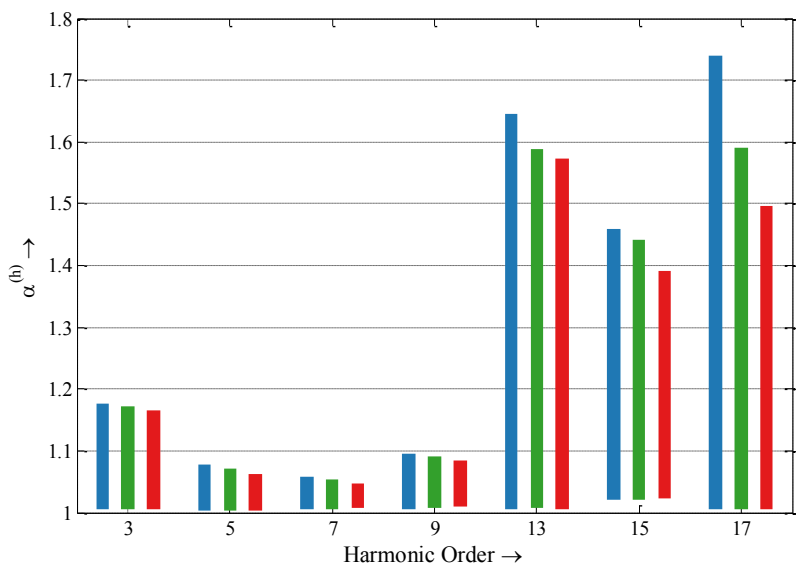

Fig. 7. Variation of diversity factor and summation exponent in phase A due to different aggregation intervals 

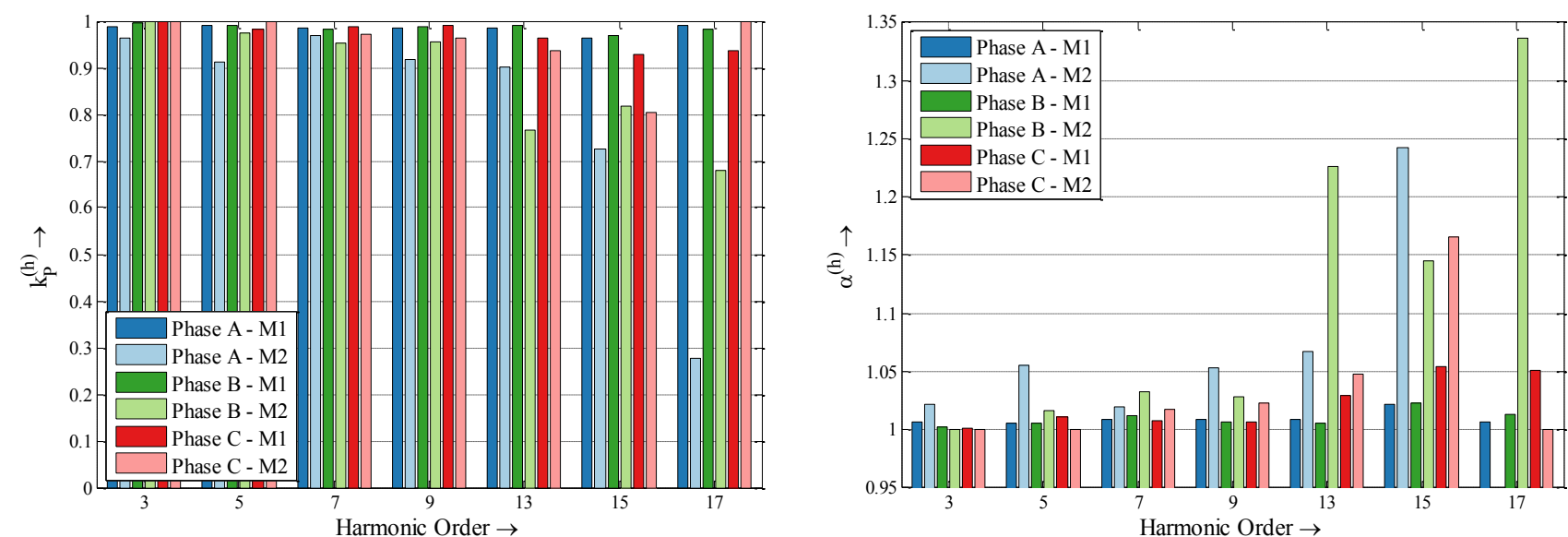

Fig.8. Diversity factor and summation exponent due to different statistical calculation methods (Method 1: M1, Method 2: M2)

\subsection{Statistical calculation method}

Finally different statistical calculation methods can be applied to find a specified percentile. In this study two methods are compared:

- Method 1: The diversity index is calculated based on the current magnitudes and phase angles in all feeders for each time instant. Then the final value is obtained as percentile from the cumulative distribution of the diversity indices for the considered observation period.

- Method 2: Cumulative distribution functions are calculated for current harmonic magnitudes and phase angles for each feeder. The final value of the diversity index is calculated based on percentiles of the cumulative distributions of harmonic magnitudes and phase angles for the considered observation period.

Fig. 8 compares the diversity indices based on method M1 and method M2. No aggregation (10-period-values) and $99^{\text {th }} / 1^{\text {st }}$ percentile for $k_{P} / \alpha$ were applied. It was not possible to calculate one summation exponent due to the high diversity of phase angles $\left(17^{\text {th }}\right.$ harmonic, phase A, M2). Once again the difference in the results is correlated to the order of the harmonic. The higher the harmonic order and subsequently the diversity, the higher is the variation between results obtained by the different methods.

Method M2 seems to result generally in lower values of the diversity factors (and higher values of $\alpha$ ), which can lead to too optimistic conclusions about the summation of harmonic currents in the network. Physically the diversity index is only defined for one single time instant. It should be noted that in method M2 the relation to time instants is lost and the meaning of the summation exponent is not clear. Therefore only method M1 can be recommended at the moment for calculation of diversity indices.

\section{Conclusion}

The presented paper studies the calculation of two commonly used diversity indices (diversity factor and summation exponent) in terms of its reliable application to time-varying harmonics. The results of the paper apply to typical consumer topologies in public LV grids with dominating share of residential users.

The use of a single measurement instrument providing magnitude and phase angle (related to the voltage fundamental of the respective phase) at high accuracy is recommended. If the use of multiple instruments is necessary, a GPS synchronization of the clocks should be used. Only in case the analysis interest is limited to harmonics below order 9, requirements on accuracy and clock synchronization could be relaxed. Beside the individual currents it is recommended to measure also the total current to enable plausibility checks. If all currents are available, the error between calculated and measured total current can be used as indicator for the accuracy and reliability of the further analysis.

The post-processing parameters aggregation interval, assessment percentile and calculation method do not show a significant impact on the harmonics with low diversity (below $9^{\text {th }}$ order for the analysed data set). For higher harmonics a higher diversity is observed and subsequently the importance of the parameters on the diversity indices increases. It should be noted that the summation exponent does not have a continuous and linear range which makes it more sensitive at higher diversities. In some cases the iterative equation may not be solved.

The calculation of diversity indices for time-varying harmonics is a complex topic. The paper represents the starting point of a systematic analysis of this issue. In the further research the focus is going to be extended to other grids.

\section{References}

[1] V. Ćuk, J. F.G. Cobben, W. L. Kling, Paulo F. Ribeiro. "Analysis of Harmonic Current Summation Based on Field Measurements". IET Generation, Transmission \& Distribution, September 2013.

[2] Blanco, A.M., Stiegler, R., Meyer, J. "Power Quality Disturbances caused by Modern Lighting Equipment (CFL and LED)". Powertech, Grenoble France, 2013.

[3] J. Meyer, P. Schegner, K. Heidenreich. "Harmonic summation effects of modern lamp technologies and small electronic household equipment," in Proc. 21st International. Conf. on Electricity Distribution (CIRED), 2011.

[4] V. Cuk, J.F.G. Cobben, W.L. Kling, R.B. Timens. "An analysis of diversity factors applied to harmonic emission limits for energy saving lamps”. ICHQP, Bergamo, 2010.

[5] Standard IEC 61000-4-30: Testing and measurement techniques - Power quality measurement methods. Ed- 3, 2012.

[6] Technical report IEC 61000-3-14. Assessment emission limits for the connection of disturbing installations to LV power systems. Ed. 1, 2011. 